Magnetohydrodynamic equilibrium and the stability of tokamak and reversed-field pinch systems with 3D helical cores

This article has been downloaded from IOPscience. Please scroll down to see the full text article. 2011 Plasma Phys. Control. Fusion 53084001

(http://iopscience.iop.org/0741-3335/53/8/084001)

View the table of contents for this issue, or go to the journal homepage for more

Download details:

IP Address: 128.178.125.164

The article was downloaded on 30/05/2011 at 07:57

Please note that terms and conditions apply. 


\title{
Magnetohydrodynamic equilibrium and the stability of tokamak and reversed-field pinch systems with 3D helical cores
}

\author{
W A Cooper ${ }^{1}$, J P Graves ${ }^{1}$, O Sauter ${ }^{1}$, D Terranova ${ }^{2}$, M Gobbin ${ }^{2}$, \\ L Marrelli ${ }^{2}, \mathbf{P}$ Martin $^{2}$ and I Predebon ${ }^{2}$ \\ ${ }^{1}$ Ecole Polytechnique Fédérale de Lausanne (EPFL), Centre de Recherches en Physique des \\ Plasmas, Association Euratom-Confédération Suisse, CH1015 Lausanne, Switzerland \\ 2 Consorzio RFX, Associazione EURATOM-ENEA sulla Fuzione, Padova, Italy \\ E-mail: wilfred.cooper@epfl.ch
}

Received 20 December 2010, in final form 7 February 2011

Published 27 May 2011

Online at stacks.iop.org/PPCF/53/084001

\begin{abstract}
Bifurcated magnetohydrodynamic (MHD) equilibrium states are computed for ITER hybrid scenario and RFX-mod SHAx configurations with very flat or reversed core magnetic shear conditions. In the ITER studies, the minimum inverse rotational transform $q_{\min }$ is near unity, while for RFX-mod it is $1 / 8$. Two equilibrium states are obtained: one is axisymmetric, the other displays a 3D helical core. In tokamak devices, the structure resembles a saturated ideal MHD internal kink mode. In the reversed-field pinch, the structure is seven-fold toroidally periodic. The equilibrium magnetic field spectrum in the Boozer coordinate frame is calculated in both the ITER and RFX-mod configurations and the implications are discussed. The RFX-mod equilibria are strongly unstable to external ideal MHD kink modes, which become stabilized with a closely fitting conducting shell when the equilibrium state has a weak reversed core shear. It is marginally unstable with a monotonic $q$-profile. Unstable modes are driven by the Ohmic current, with pressure and PfirschSchlüter currents having a very weak effect. The external kink mode spectrum is dominated by coupled $m=1, n=6$ and $m=2, n=13$ Fourier components, which revert to $m=1, n=8$ and $m=2, n=15$ terms with a conducting wall in proximity to the plasma-vacuum interface.
\end{abstract}

(Some figures in this article are in colour only in the electronic version)

\section{Introduction}

Tokamaks and reversed field pinches (RFPs) are essentially axisymmetric devices. However, the Single Helical Axis (SHAx) state observed in RFX-mod [1], snake structures in the JET tokamak [2], the disappearance of sawteeth but continuous dominant $n=1$ modes seen in 
TCV at high elongation and current $[3,4]$, the change in sawteeth from kink-like to quasiinterchange-like reported on DIII-D [5], the long-lived saturated modes in MAST [6] and the saturated internal kinks observed on NSTX [7], constitute clear manifestations of the existence of magnetohydrodynamic (MHD) equilibrium states that break the axisymmetric conditions imposed by the external coils. These quasi-stationary states have a three-dimensional (3D) character that cannot be reproduced with standard Grad-Shafranov MHD solvers.

Analytic models of internal helical states in tokamaks have described saturated ideal MHD internal kinks [8-10]. Pellet deposition inside the rational $q=1$ magnetic island has been conjectured as an explanation for the snake phenomena in JET [11]. Large scale nonlinear MHD stability codes are routinely used to obtain saturated conditions that can closely model the experimental conditions [12-14]. A second variation of the potential energy has been employed to calculate neighbouring equilibrium states with 3D structures interpreted as indicators of magnetic island formation [15].

We are motivated to investigate the proposition that the saturated instability structures observed in the experiments we have alluded to, constitute in reality novel MHD equilibrium states with 3D character. To preclude that the structures that are computed are linked to or are a direct consequence of any possible 3D perturbation applied to the plasma-vacuum interface, we impose exact fixed axisymmetric boundaries.

A 3D MHD equilibrium solver must be applied for this purpose. The most convenient and fastest tool currently available is the VMEC code [16,17]. The equilibrium calculations for RFX-mod that we shall report in this paper are performed with the version of VMEC in which the toroidal magnetic flux is the independent radial variable, which implies that toroidal magnetic field reversal can only be approached. For the core physics phenomena we are attempting to model, this limitation is, for all practical purposes, inconsequential for equilibrium computations but could possibly alter the stability analysis. Initial RFX-mod SHAx equilibrium state simulations in which seven-fold periodic 3D core helical structures develop have been published [18]. Bifurcated solutions of tokamak MHD equilibria are successfully obtained with the ANIMEC code (an anisotropic pressure version of VMEC, although scalar pressure is imposed in the simulations undertaken) [19]. Two solutions can spontaneously develop, but only the axisymmetric result can be calculated with a GradShafranov solver. The second solution corresponds to a $3 \mathrm{D}$ internal helical core structure that looks like a saturated ideal MHD $m=1, n=1$ kink mode. MHD equilibria of this type have been calculated for TCV configurations with high current and elongation [20], for ITER hybrid scenario [21] configurations [22] and for the MAST tokamak [23]. The linear ideal MHD stability properties of 3D configurations are investigated with the TERPSICHORE code [24]. We specifically concentrate on instabilities that break the periodicity of the underlying equilibrium state. We have previously examined the stability of RFX-mod SHAx equilibria with a conducting wall at a position similar to that in the experiment [23].

We extended the work that we previously carried out in ITER hybrid scenario equilibria [22] and the RFX-mod linear ideal MHD stability [23] by, respectively, analysing the bifurcated axisymmetric and 3D helical core equilibrium states in Boozer magnetic coordinates [25], and the effects of conducting wall positioning on RFX-mod stability. The transformation of the equilibrium state for the tokamak and the RFP equilibria from the coordinates of the VMEC code to the Boozer frame are performed with internal modules of the TERPSICHORE code [24] and are necessary for the evaluation of MHD stability and guiding centre particle orbits for neoclassical transport studies. Advanced stellarator systems with improved neoclassical confinement have been devised, based in a major part on the optimization of the mod- $B$ spectrum in these coordinates [26,27], and insights into particle confinement can be gleaned from an analysis of this spectrum. 


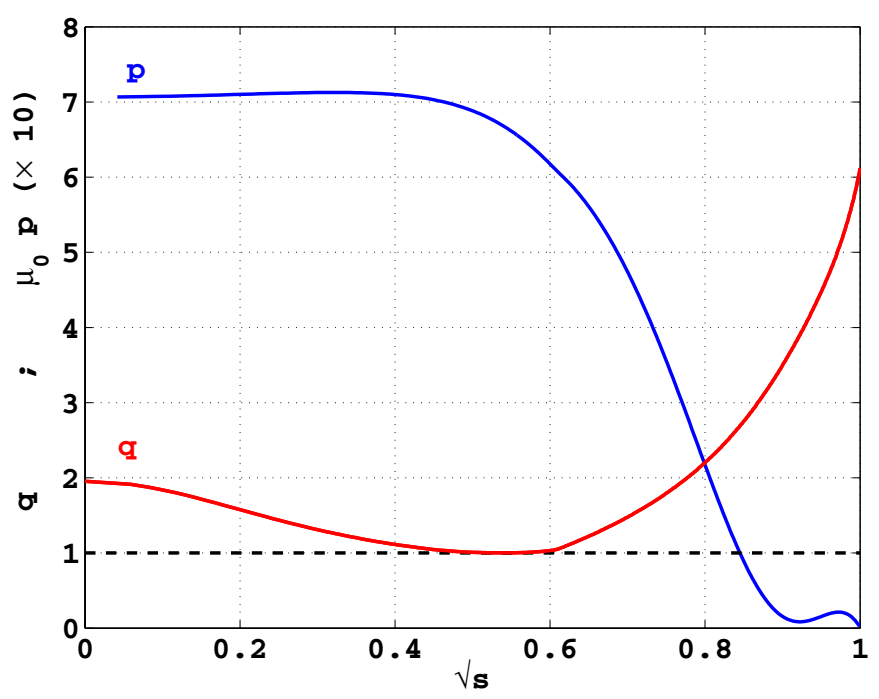

Figure 1. The pressure and inverse rotational transform $q$-profiles as a function of $\sqrt{s}$, where the radial variable $s(0 \leqslant s \leqslant 1)$ is proportional to the enclosed toroidal magnetic flux. The shape of the $q$-profile is characteristic of an ITER hybrid scenario case. Note that the pressure is multiplied by $10 \mu_{0}$ with $\mu_{0}=4 \pi \times 10^{-7} \mathrm{H} \mathrm{m}^{-1}$.

In section 2, we investigate a hybrid scenario 3D helical ITER equilibrium state in the Boozer magnetic coordinate system. In section 3, we examine the equilibrium and the global linear ideal MHD stability of an RFX-mod SHAx state. Conclusions and a discussion are presented in section 4 .

\section{ITER hybrid scenario equilibrium state in the Boozer coordinates}

In this section, we explore an ITER equilibrium state bifurcation in the Boozer coordinate frame. We have previously generated a sequence of ITER equilibria and examined the 3D helical core states as functions of the minimum value of the inverse rotational transform $q_{\text {min }}$ and of $\langle\beta\rangle$ [22]. Here we concentrate on axisymmetric and helical branches of a configuration at $\langle\beta\rangle \simeq 2.79 \%$, with $q_{\text {min }} \sim 1$ and $12 \mathrm{MA}$ toroidal current. The pressure and $q$-profile are displayed in figure 1 . To provide the appropriate background for comparison, the toroidal magnetic flux function $\Phi$ and the mod- $B$ contours of the axisymmetric branch equilibrium state in the Boozer coordinates are presented in figure 2. The corresponding contours for the helical branch on four cross sections that span half the torus show that the constant $\Phi$ contours develop a 3D core embedded within a virtually axisymmetric mantle, but that the constant mod- $B$ lines are not modified significantly from the axisymmetric solution. These results are presented in figure 3. A visual examination would indicate that particles in the core of the plasma could develop super-banana orbits which tend to be poorly confined. If they drift into the axisymmetric domain, they would become normal trapped particles and their confinement properties would improve. The Fourier spectrum of $B^{2}$ for the bifurcated equilibrium states appear in figure 4 . The axisymmetric branch has only toroidal mode number $n=0$ components. The helical branch shows that in the plasma a toroidal $m=1, n=0$ term competes with a $m=0, n=1$ mirror term. The poloidal mode number is $m$. Near the minimum value of $q$, a helical $m=2, n=1$ term becomes important. Towards the edge of 

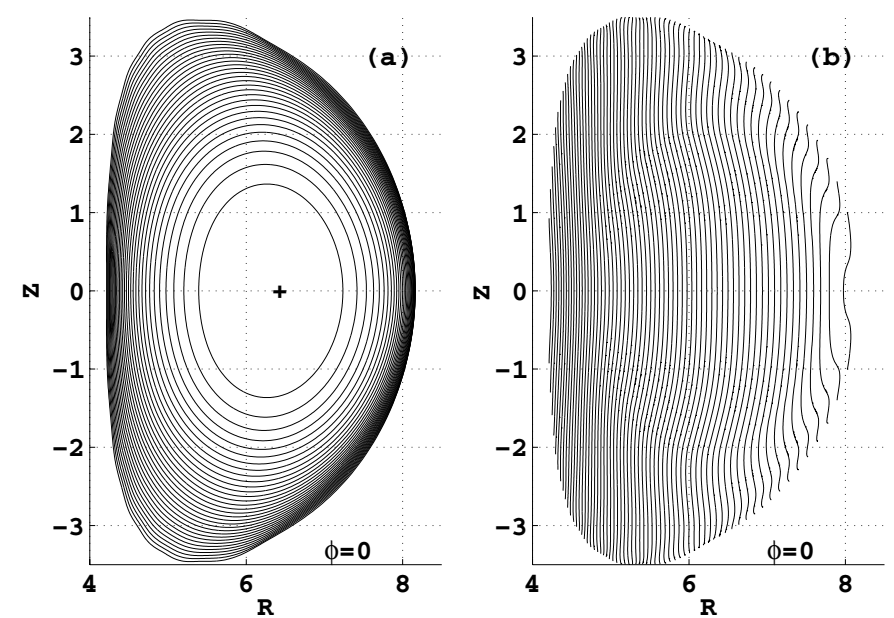

Figure 2. The toroidal magnetic flux (left) and mod- $B$ (right) contours of the axisymmetric branch of an ITER hybrid scenario equilibrium state in the Boozer magnetic coordinates.

the plasma, the nonaxisymmetric mod- $B^{2}$ Fourier components vanish, so particle behaviour similar to that of a conventional tokamak can be anticipated in that region.

\section{Wall stabilization of ideal kink modes in RFX-mod}

MHD equilibrium states that model the SHAx regime on RFX-mod configurations are obtained with the VMEC code [17] and have been reported in a recent publication [18]. The inverse rotational transform for equilibrium states with monotonic $q$-profiles and with reversed core magnetic shear appear in figure 5. In figure 6, in analogy with the ITER equilibria analysed in the previous section, we present the toroidal magnetic flux function $\Phi$ contours and the corresponding mod- $B$ distribution at four cross sections covering half of a toroidal field period (in the seven-fold periodic system) in Boozer magnetic coordinates for the reversed core shear case. The flux contours show a clear helical deformation of the plasma core. The mod- $B$ distribution demonstrates the maximum- $B$ characteristics of the RFP where the $B$-strength is maximum on axis. The mod- $B$ distribution does not change significantly with toroidal angle. However, from a guiding centre particle drift perspective, we could anticipate super-banana formation in the central region of the plasma. The Fourier spectrum of $B^{2}$ in the Boozer frame shows a dominant $m=0, n=0$ component as illustrated in figure 7 . In the bulk of the plasma, toroidal, mirror and helical terms are all relevant, however, only $n=0$ Fourier components survive towards the edge of the plasma, so particles that drift into this region could be expected to become better confined. Neoclassical transport calculations in force-free equilibria with reconstructed magnetic perturbations [28] agree with this assertion.

The linear ideal MHD stability analysis of RFX-mod SHAx equilibria is computed with the TERPSICHORE code [24], which evaluates the variational energy principle

$$
\delta W_{\mathrm{P}}+\delta W_{\mathrm{V}}-\omega^{2} \delta W_{\mathrm{K}}=0 .
$$

The internal potential energy is

$$
\delta W_{\mathrm{P}}=\frac{1}{2} \iiint \mathrm{d}^{3} x\left[C^{2}+\Gamma p|\nabla \cdot \boldsymbol{\xi}|^{2}-D|\boldsymbol{\xi} \cdot \nabla s|^{2}\right] .
$$



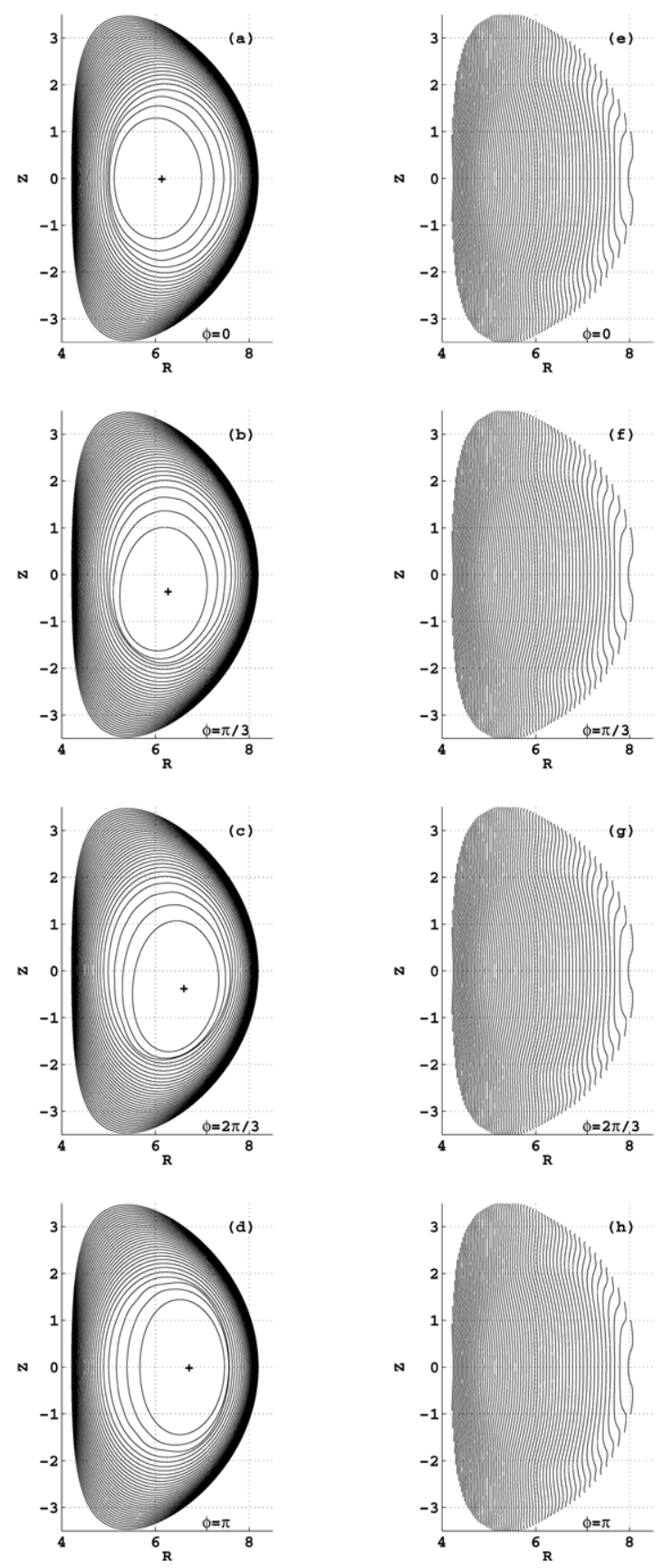

Figure 3. The toroidal magnetic flux $\Phi$ contours (left column) and the mod- $B$ lines (right column) at cross sections with the Boozer toroidal angle $\phi=0, \phi=\pi / 3, \phi=2 \pi / 3$ and $\phi=\pi$ (from top to bottom) in the $3 \mathrm{D}$ helical core branch of an ITER hybrid scenario equilibrium state.

The stabilizing mainly perturbed magnetic field term is expressed as

$$
\boldsymbol{C}=\nabla \times(\xi \times B)+\frac{j \times \nabla s}{|\nabla s|^{2}}(\xi \cdot \nabla s)
$$



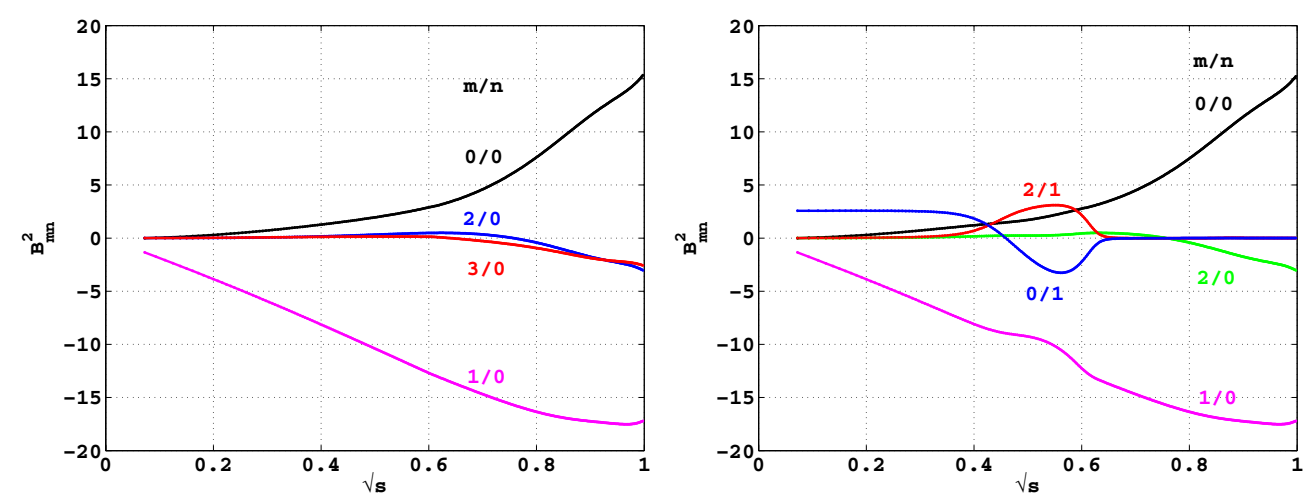

Figure 4. The Fourier amplitudes of the profiles of the dominant components of $B^{2}$ in the Boozer coordinates plotted as a function of $\sqrt{s}$ in the ITER hybrid scenario axisymmetric equilibrium branch (left) and 3D helical equilibrium branch (right).

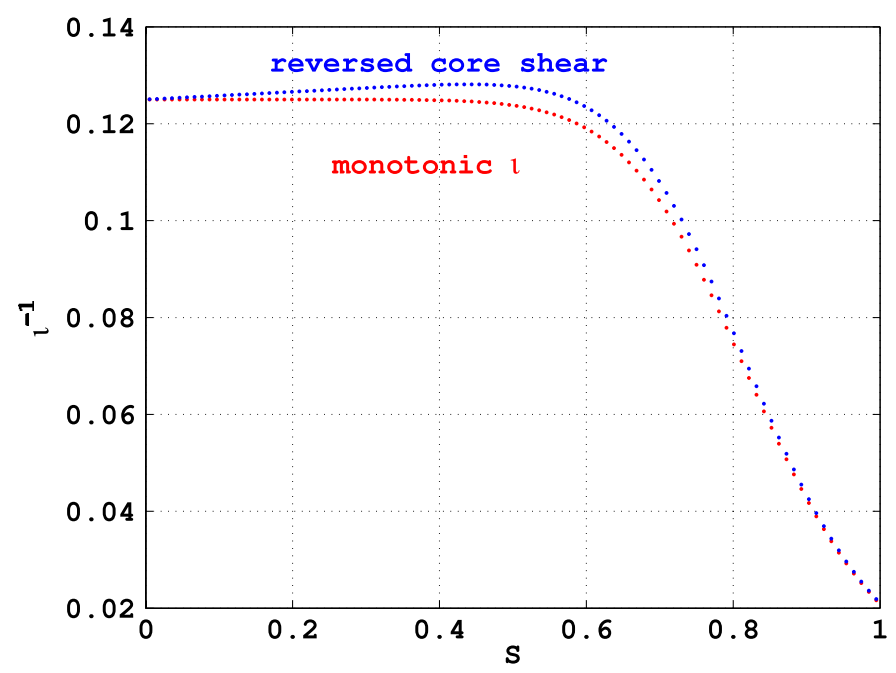

Figure 5. The inverse rotational transform $1 / \iota=q$ profiles for RFX-mod SHAx equilibrium states with a monotonic $q$-profile and $q^{\prime} / q<0$ throughout the plasma, and with reversed core magnetic shear $q^{\prime} / q>0$ in the centre of the plasma.

where $\boldsymbol{\xi}$ is the MHD fluid displacement vector and $j$ is the equilibrium current density. A model kinetic energy is invoked that annihilates the parallel component of $\boldsymbol{\xi}$ in TERPSICHORE, hence the plasma compressibility term in $\delta W_{\mathrm{P}}$ can be analytically eliminated [29]. That leaves the instability driving term $D$ given by

$$
D=\frac{2(j \times \nabla s) \cdot(B \cdot \nabla) \nabla s}{|\nabla s|^{4}} .
$$

The vacuum energy is

$$
\delta W_{\mathrm{V}}=\frac{1}{2} \iiint \mathrm{d}^{3} x|\nabla \times \boldsymbol{A}|^{2} .
$$

A pseudoplasma vacuum domain is constructed in TERPSICHORE that allows $\delta W_{\mathrm{V}}$ to be expressed in a structure very similar to that of $\delta W_{\mathrm{P}}$ [29]. A conducting wall is then prescribed that can closely approach the plasma-vacuum interface. 

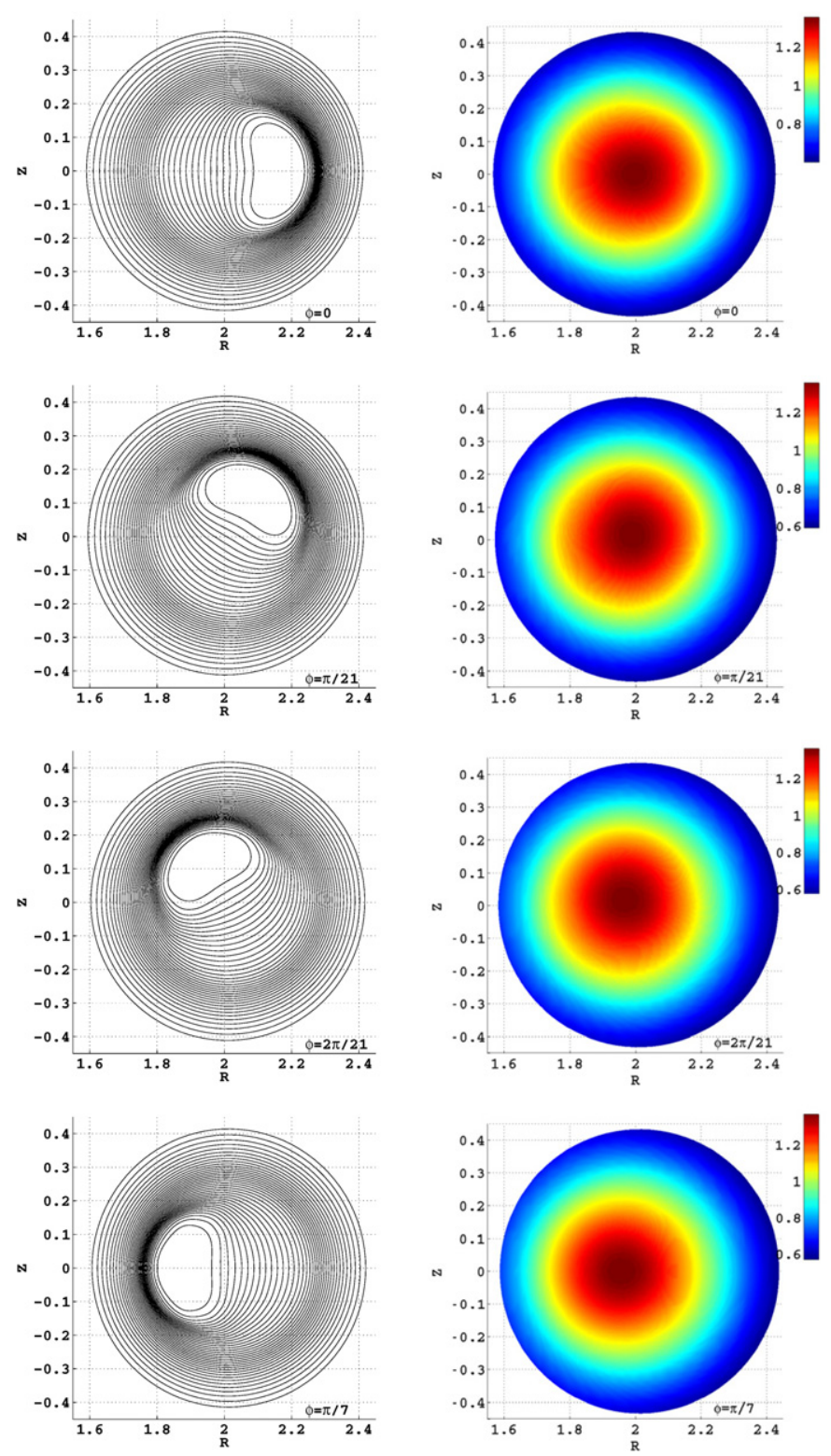

Figure 6. The toroidal magnetic flux $\Phi$ contours (left column) and the mod- $B$ distribution (right column) at cross sections with the Boozer toroidal angle $\phi=0, \phi=\pi / 21, \phi=2 \pi / 21$ and $\phi=\pi / 7$ (from top to bottom) for an RFX-mod SHAx equilibrium state with reversed core magnetic shear.

In MHD stability analysis, the requirement for a very precise description of the $\boldsymbol{B} \cdot \boldsymbol{\nabla}$ operator favours the utilization of straight magnetic field line coordinates. Of the infinite class of these type of coordinates, the Boozer frame is preferred because the poloidal and toroidal magnetic field components in the covariant representation correspond to the toroidal and poloidal current flux functions $J$ and $I$, respectively. As these quantities are flux surface functions, complicated algebraic manipulations are simplified immensely. We express the 


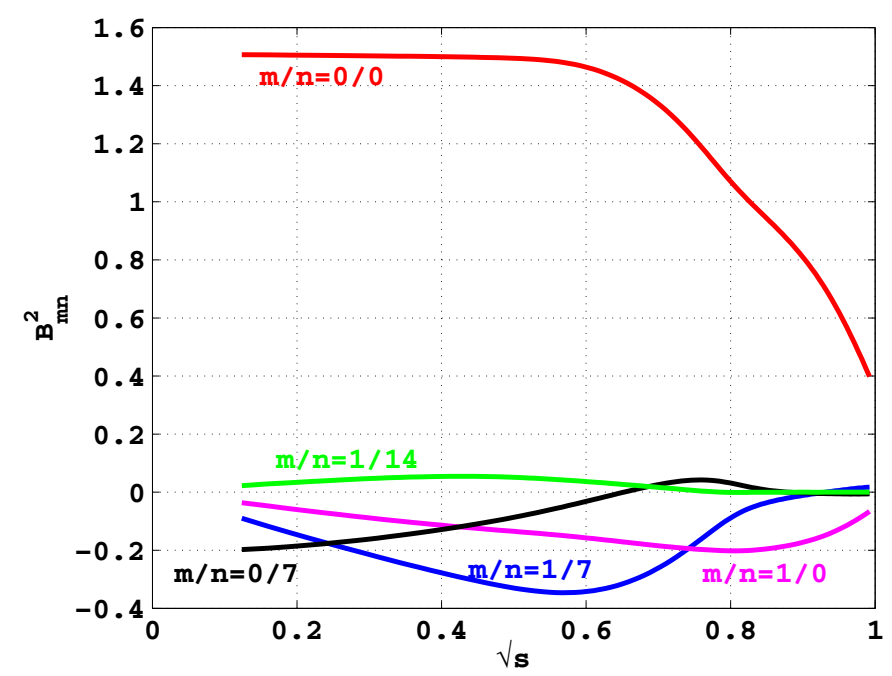

Figure 7. The Fourier amplitude profiles for the five dominant components of $B^{2}$ in the Boozer coordinates plotted as a function of $\sqrt{s}$. The radial variable $s$ corresponds to the normalized toroidal magnetic flux for the reversed core shear RFX-mod SHAx equilibrium case.

displacement vector as

$$
\boldsymbol{\xi}=\sqrt{g} \xi^{s}(\nabla \theta \times \nabla \phi)+\frac{\boldsymbol{B} \times \nabla s}{B^{2}} \eta+\left[\frac{J(s)}{\Phi^{\prime}(s) B^{2}} \eta-\mu\right] \boldsymbol{B},
$$

where $\theta$ and $\phi$ are the Boozer coordinate poloidal and toroidal angles, respectively, $\sqrt{g}$ denotes the Jacobian and prime (') indicates the derivative of a flux surface quantity with respect to $s$.

Fourier decomposition in the angular variable is applied. Thus we write

$$
\begin{aligned}
\xi^{s}(s, \theta, \phi) & =\sum_{\ell} s^{-q_{\ell}} X_{\ell}(s) \sin \left(m_{\ell} \theta-n_{\ell} \phi+\Delta\right), \\
\eta(s, \theta, \phi) & =\sum_{\ell} Y_{\ell}(s) \cos \left(m_{\ell} \theta-n_{\ell} \phi+\Delta\right),
\end{aligned}
$$

where $\ell$ identifies a $(m, n)$ mode pair and $\Delta$ is a phase factor (either 0 or $\pi / 2)$ associated with the even or odd parity of the mode structure [30]. The radial discretization is performed with COOL finite elements [31]. The radial domain is decomposed as

$$
s=s_{j-\frac{1}{2}}+\frac{\Delta s}{2} r ; \quad-1 \leqslant r \leqslant 1,
$$

hence $r=2 /(\Delta s)\left(s-s_{j-1 / 2}\right)$. Hybrid finite element basis functions based on Legendre polynomials are invoked at each interval, centred about $s_{j-1 / 2}$ with index $1 \leqslant j \leqslant N_{r}$ where $N_{r}$ is the total number of radial intervals. The basis functions are

$$
\begin{aligned}
h_{i}(r) & =\beta_{i} \frac{\left(1-r^{2}\right) L_{p}(r)}{\left(r-\zeta_{1}\right)\left(r-r_{i}\right)}, \\
g_{i}(r) & =\gamma_{i} \frac{L_{p}(r)}{\left(r-\zeta_{i}\right)},
\end{aligned}
$$

where $L_{p}(r)$ is the Legendre polynomial of order $p, \zeta_{i}$ identifies the Gauss points (the zeroes of the Legendre polynomial) and $r_{i}$ corresponds to a value of $-1, \zeta_{i} \neq \zeta_{1}$ or 1 . $\beta_{i}$ and $\gamma_{i}$ are 

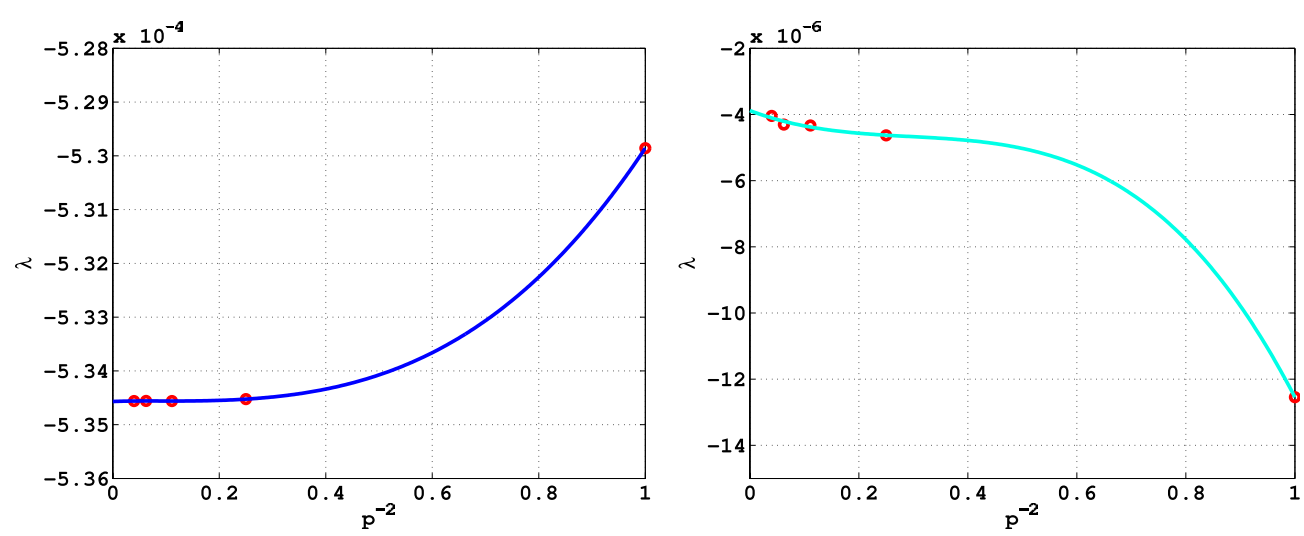

Figure 8. Convergence of the unstable eigenvalue as a function of $1 / p^{2}$, where $p$ is the order of the Legendre polynomial in the basis function used for the radial discretization. The solutions presented are for the monotonic $q$-profile (left) and for the core shear reversed (right) RFX-mod SHAx equilibria.

normalization factors. Thus, in the radial domain $s_{j-1} \leqslant s \leqslant s_{j}$, we can write

$$
\begin{aligned}
& X_{\ell}(s) \simeq h_{1}(r) X_{\ell}^{j-1}+\sum_{i=2}^{p} h_{i}(r) X_{\ell}\left(s\left(r=\zeta_{i}\right)\right)+h_{p+1}(r) X_{\ell}^{j}, \\
& Y_{\ell}(s)=\sum_{i=1}^{p} g_{i}(r) Y_{\ell}\left(s\left(r=\zeta_{i}\right)\right) .
\end{aligned}
$$

The choice $p=1$ recovers the standard tent and hat function discretization for $X$ and $Y$, respectively. With this description, the variational ideal MHD stability equation reduces to a special block pentadiagonal matrix eigenvalue equation that is solved with an inverse iteration technique. One very useful feature of the discretization method we have implemented is that we can perform convergence studies with respect to the radial mesh size without modifying the underlying equilibrium by changing the order $p$ of the Legendre polynomial. This is shown specifically (for the two equilibrium states we investigate whose $q$-profiles were presented in figure 5) in figure 8 , where the eigenvalue $\lambda=-\omega^{2}$ is plotted as a function of $1 / p^{2}$ with $p \geqslant 2$, demonstrating very good convergence when $N_{r}=96$.

The ideal MHD stability of RFX-mod SHAx equilibrium states is carried out with respect to mode family structures that break the seven-fold periodicity of the system. We specifically look for toroidal sideband modes that are adjacent to multiples of seven. Thus we examine structures with toroidal mode number $n=k n_{\mathrm{eq}} \pm 1$, where $n_{\mathrm{eq}}=7$ and $k$ is any integer. Thus the toroidal mode numbers fall in the category $(n=\ldots-1,1,6,8,13,15,20 \ldots)[32,33]$. The unstable eigenvalues versus wall position for the monotonic $q$ and for the reversed core shear $q$-profile cases are displayed in figure 9. A conforming conducting wall is prescribed. The eigenvalue $\lambda$ is plotted with respect to $w / a$ and the ratio of the wall to the plasma diameter at the midplane. The plasma is unstable in both cases with the wall far from the plasma, but becomes strongly stabilized when $w / a \leqslant 1.2$. In the RFX-mod experiment $w / a \simeq 1.1$, and we have investigated this case in more detail previously [23]. For the monotonic $q$-profile case, the plasma remains marginally unstable $\left(\lambda \sim-4.4 \times 10^{-4}\right)$ for $w / a \leqslant 1.2$. For the reversed core shear case $\left(q^{\prime} / q>0\right)$, the eigenvalue $\lambda$ is about $-4 \times 10^{-6}$. The model kinetic energy we have invoked that annihilates the parallel component of $\boldsymbol{\xi}$ implies that the eigenvalue $\lambda$ does not represent the square of a physical growth rate (normalized, for example, to the Alfvén 

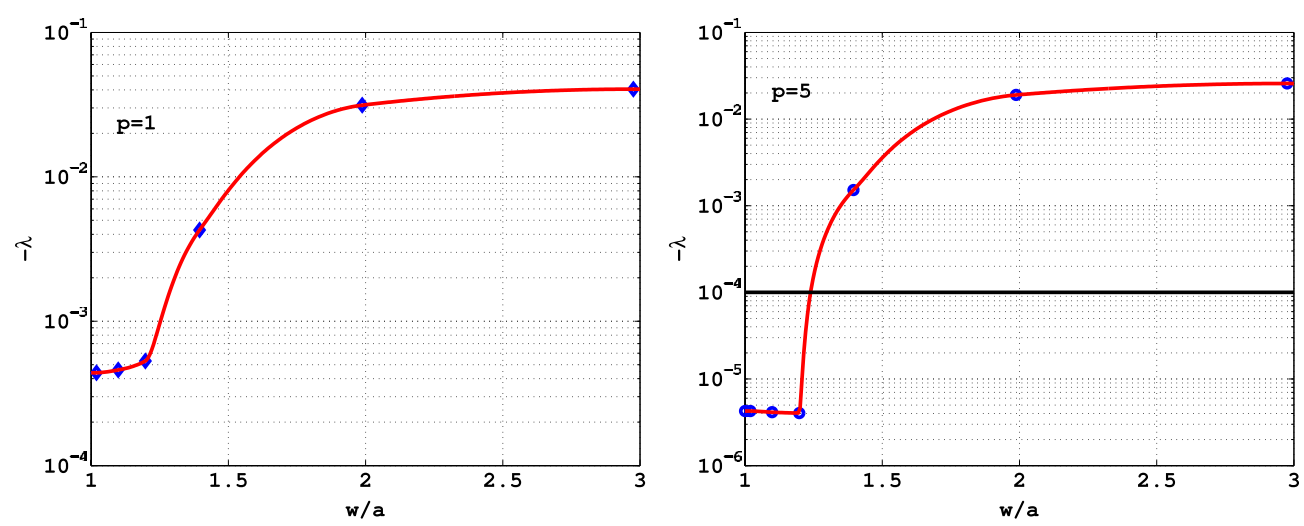

Figure 9. The unstable eigenvalue $\lambda$ as a function of the conducting wall position $w / a$ in RFX-mod SHAx equilibria with monotonic $q$-profile $\left(q^{\prime} / q<0\right.$ across the plasma) (left) and with reversed core magnetic shear $\left(q^{\prime} / q>0\right.$ in the centre of the plasma) (right).
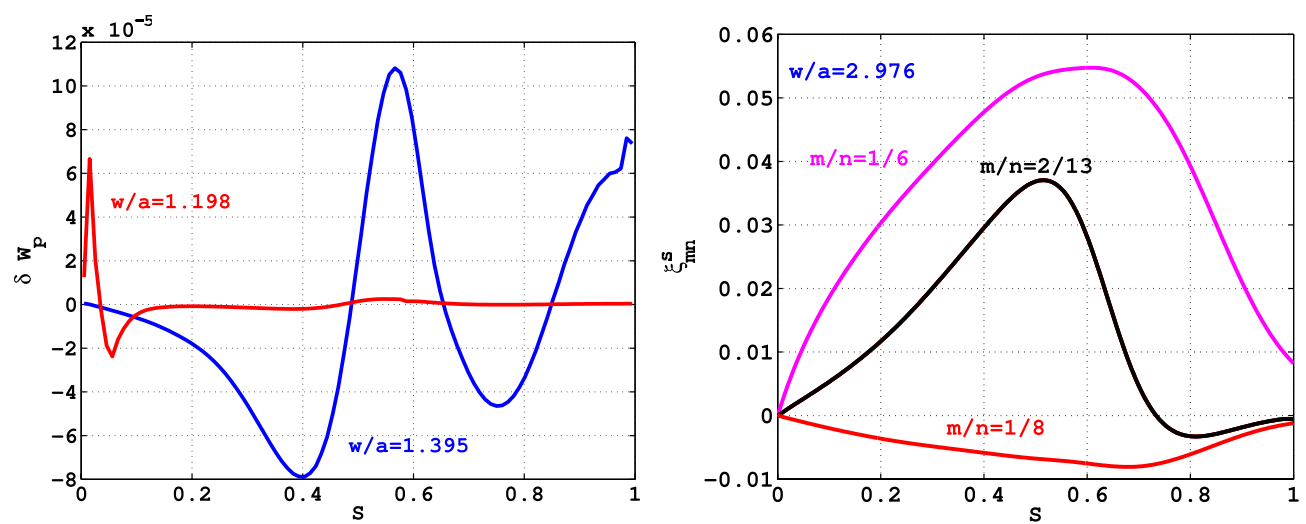

Figure 10. The potential energy $\delta W_{\mathrm{P}}$ profiles for $w / a=1.198$ and 1.395 (left) and the three leading Fourier amplitudes of the radial component of the displacement vector (right) for $w / a=2.976$ with reversed core shear $\left(q^{\prime} / q>0\right.$ on axis).

frequency). Previous applications of TERPSICHORE mostly on stellarator configurations indicate that a threshold value of $\lambda=-1 \times 10^{-4}$ constitutes a representative level for marginal stability. Eigenvalues that are more negative than this critical value are considered to be unstable. In figure 10, we plot the $\delta W_{\mathrm{P}}$ radial profiles for $w / a \sim 1.2$ and $w / a \sim 1.4$. As the wall approaches the plasma-vacuum interface, the mode tends to become localized in the core with $\lambda$ within the marginally stable range when $q^{\prime} / q>0$ on axis. The Fourier amplitudes of the radial component of the displacement vector profiles with the conducting wall far from the plasma are also displayed in this figure. The mode structure is dominated by the $m=1, n=6$ term strongly coupled with the $m=2, n=13$ term (a different instability branch sets in with a closely fitting conducting shell where a $m=1, n=8$ coupled to a $m=2, n=15$ mode becomes dominant [23]).

The instability drive term $D$ can be expanded and separated into two parts $[34,35]$. The term associated with ballooning-interchange modes in which the pressure gradient interacts 

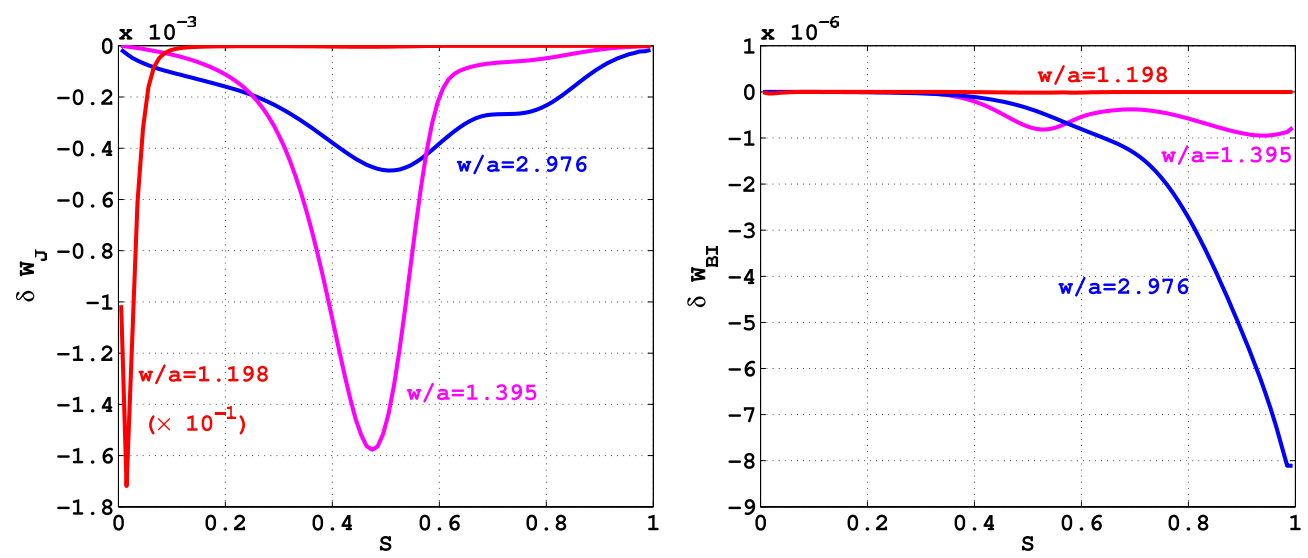

Figure 11. The profiles of $\delta W_{J}$ (left) and $\delta W_{\mathrm{BI}}$ (right) for wall positions $w / a=1.198$, $w / a=1.395$ and $w / a=2.976$ in an RFX-mod SHAx equilibrium with reversed central magnetic shear $\left(q^{\prime} / q>0\right)$.

with the magnetic field line curvature can be written as

$$
\begin{gathered}
\delta W_{\mathrm{BI}}=-\frac{1}{2} \int_{0}^{1} \mathrm{~d} s \int_{0}^{\frac{2 \pi}{L_{s}}} \mathrm{~d} \phi \int_{0}^{2 \pi} \mathrm{d} \theta p^{\prime}(s)\left[\frac{\sqrt{g} p^{\prime}(s)+\psi^{\prime \prime}(s) J(s)-\Phi^{\prime \prime}(s) I(s)}{B^{2}}-\frac{\partial \sqrt{g}}{\partial s}\right]\left(\xi^{s}\right)^{2} \\
+\frac{1}{2} \int_{0}^{1} \mathrm{~d} s \int_{0}^{\frac{2 \pi}{L_{s}}} \mathrm{~d} \phi \int_{0}^{2 \pi} \mathrm{d} \theta p^{\prime}(s) \frac{B_{s}}{B^{2}} \xi^{s}\left(\sqrt{g} \boldsymbol{B} \cdot \nabla \xi^{s}\right),
\end{gathered}
$$

where $L_{s}$ is the number of equilibrium field periods per stability structure period (in our case $L_{s}=7$ ) and $B_{s}$ is the radial component of the magnetic field in the covariant representation. The term associated with kink mode stability corresponds to the interaction of the parallel current density with the local magnetic shear and can be expressed as

$$
\begin{aligned}
\delta W_{J}= & -\frac{1}{2} \int_{0}^{1} \mathrm{~d} s \int_{0}^{\frac{2 \pi}{L_{s}}} \mathrm{~d} \phi \int_{0}^{2 \pi} \mathrm{d} \theta\left[\frac{\sqrt{g} B^{2}}{|\nabla s|^{2}}\left(\frac{\boldsymbol{j} \cdot \boldsymbol{B}}{B^{2}}\right)+\psi^{\prime}(s) \Phi^{\prime \prime}(s)\right. \\
& \left.-\Phi^{\prime}(s) \psi^{\prime \prime}(s)\right]\left(\frac{\boldsymbol{j} \cdot \boldsymbol{B}}{B^{2}}\right)\left(\xi^{s}\right)^{2} \\
& +\frac{1}{2} \int_{0}^{1} \mathrm{~d} s \int_{0}^{\frac{2 \pi}{L_{s}}} \mathrm{~d} \phi \int_{0}^{2 \pi} \mathrm{d} \theta\left(\frac{\boldsymbol{j} \cdot \boldsymbol{B}}{B^{2}}\right) h_{s} \xi^{s}\left(\sqrt{g} \boldsymbol{B} \cdot \nabla \xi^{s}\right),
\end{aligned}
$$

where the residual local magnetic shear is

$$
h_{s}=-\frac{1}{|\nabla s|^{2}}\left[I(s) \frac{g_{s \theta}}{\sqrt{g}}+J(s) \frac{g_{s \phi}}{\sqrt{g}}\right] .
$$

In figure 11, we show the radial profiles of $\delta W_{J}$ and $\delta W_{\mathrm{BI}}$ for three wall positions $(w / a \sim 1.2$, $w / a \sim 1.4$ and $w / a \sim 3.0$ ). The mode is dominantly a kink mode as $\delta W_{J}$ is two to three orders of magnitude larger than $\delta W_{\mathrm{BI}}$. The mode becomes more localized and squeezed towards the plasma core with shrinking wall dimensions. Finally, the drive for the instability is the Ohmic current. The Pfirsch-Schlüter currents, which correspond to the flux surface varying components of the parallel current density $\boldsymbol{j} \cdot \boldsymbol{B} / \boldsymbol{B}^{2}$ and are proportional to the pressure gradient, contribute very weakly to the mode. The structure of the kink mode driving energy 

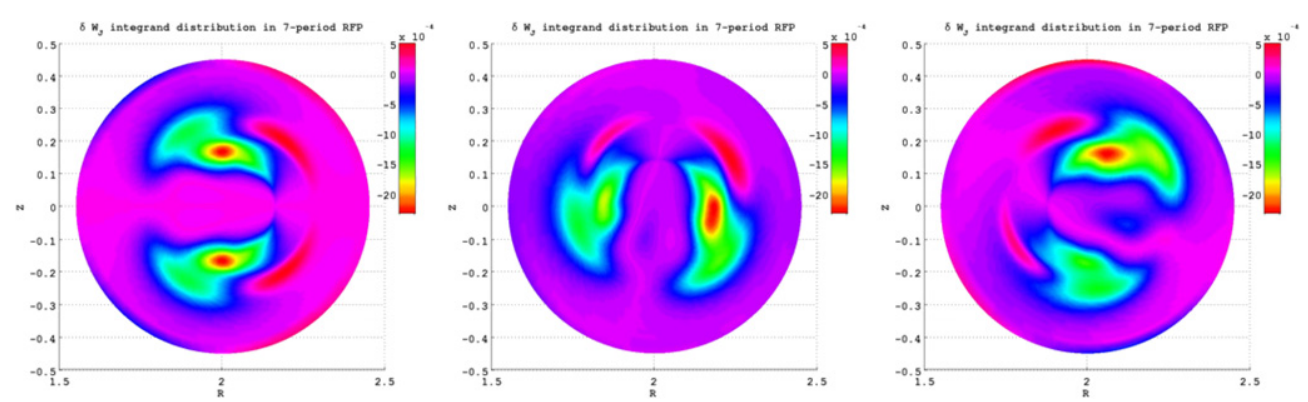

Figure 12. The $\delta W_{J}$ distribution at the $\phi=0$ (left), $\phi=\pi / 14$ (middle) and $\phi=\pi / 7$ (right) Boozer toroidal angle cross sections in an RFX-mod SHAx equilibrium state with a conducting wall far from the plasma $(w / a=2.976)$ and a monotonic $q$-profile with $q^{\prime} / q<0$ everywhere.

$\delta W_{J}$ for $q^{\prime} / q<0$ (monotonic $q$-profile) with the conducting wall far from the plasma on three different cross sections are shown in figure 12 .

\section{Conclusions and discussion}

We have generated MHD equilibria that model the ITER hybrid scenario with a fixed axisymmetric boundary and demonstrated that bifurcated states exist. The standard state is axisymmetric. The second state has a $3 \mathrm{D}$ internal helical core that resembles a saturated $m=1, n=1$ internal ideal kink mode which cannot be computed with standard GradShafranov equilibrium solvers. The primary feature for the spontaneous bifurcation that is observed corresponds to the shape of the $q$-profile. Profiles with weak core reversed magnetic shear and $q_{\text {min }}$ in the neighbourhood of unity, which are typical for the ITER hybrid scenario, are susceptible to develop two solutions. The effects of the pressure profile shape and $\langle\beta\rangle$ are secondary. The global MHD stability of the helical configurations must be evaluated with great care as the same modes that describe the equilibrium state must be applied for stability analysis. This shall be explored in future work.

MHD equilibria that model the RFX-mod SHAx configurations that approach toroidal magnetic field reversal at the edge of the plasma have been investigated. We concentrate in this paper on wall stabilization effects on global linear ideal MHD instabilities. The equilibria we examine in detail have either flat monotonic core shear or weak central reversed shear. We have specifically looked at mode families with sideband toroidal mode numbers that are immediately adjacent to multiples of seven (the equilibrium has seven-fold toroidal periodicity). With a conducting wall far from the plasma, both configurations investigated are strongly unstable to a global ideal MHD kink mode dominated by a $m=1, n=6$ Fourier component coupled with a $m=2, n=13$ sideband. The ballooning-interchange source of free energy instability is very weak, thus the mode is primarily driven by the Ohmic current (the Pfirsch-Schlüter current is also small). These kink modes are effectively stabilized by a conducting wall, which suppresses the ( $m=1, n=6 ; m=2, n=13$ ) components. The residual mode that remains couples finite $m=1, n=8$ and $m=2, n=15$ amplitudes. The plasma is weakly unstable for monotonic central $q^{\prime} / q<0$ profiles when the conducting wall to plasma midplane diameter is $w / a \leqslant 1.2$, but becomes virtually stable for the reversed core magnetic shear $\left(q^{\prime} / q>0\right)$ as the eigenvalue $\lambda$ falls within the stability threshold $\lambda_{\text {thresh }}=-1 \times 10^{-4}$. Toroidal sideband coupling constitutes a significant contribution. In RFX-mod, the SHAx state periodically relaxes [36], and ideal MHD modes are conjectured as a possible cause. 
The principal shortcoming of the stability analysis undertaken in this work was related to RFP equilibria without toroidal magnetic field reversal. Although the ideal MHD computations carried out included $m=0$ mode components, these were nonresonant. The implications of the coupling of resonant $m=0$ mode terms with the dominant $m=1$ kink structures in the presence of toroidal magnetic field reversal must still be determined in the SHAx RFXmod configurations. An extension of the 3D VMEC equilibrium code in which the poloidal magnetic flux is employed as the radial coordinate will allow future studies on this subject.

The magnetic field strength Fourier amplitude profiles in both ITER and RFX-mod SHAx 3D helical core equilibrium states both demonstrate that the central region of the plasma combines axisymmetric, mirror and helical components, while the nonaxisymmetric components in the edge of the plasma vanish. This suggests that the neoclassical transport properties should be poor in the centre of the plasma (similar to conventional stellarators), and then recover the axisymmetric limit at the helical to toroidal transition layer. This could then lead to the appearance of a transport barrier just outside this layer [37, 38].

\section{Acknowledgments}

We thank S P Hirshman for the development and use of the VMEC code package on which the work presented in this paper has relied. This research was partially sponsored by the Fonds National Suisse de la Recherche Scientifique and by Euratom. The views and opinions expressed herein do not necessarily reflect those of the European Commission.

Euratom (C) 2011.

\section{References}

[1] Lorenzini R et al 2009 Nature Phys. 5570

[2] Weller A et al 1987 Phys. Rev. Lett. 592303

[3] Reimerdes H et al 2006 Plasma Phys. Control. Fusion 481621

[4] Camenen Y et al 2007 Nucl. Fusion 47586

[5] Lazarus E A et al 2006 Plasma Phys. Control. Fusion 48 L65

[6] Chapman I T et al 2010 Nucl. Fusion 50045007

[7] Menard J E et al 2005 Nucl. Fusion 45539

[8] Avinash, Hastie R J, Taylor J B and Cowley S C 1987 Phys. Rev. Lett. 592647

[9] Bussac M N and Pellat R 1987 Phys. Rev. Lett. 592650

[10] Waelbrock F L et al 1989 Phys. Fluids B 1499

[11] Wesson J A 1995 Plasma Phys. Control. Fusion 37 A337

[12] Charlton L A, Hastie R J and Hender T C 1989 Phys. Fluids B 1798

[13] Lütjens H and Luciani J F 2008 J. Comput. Phys. 2276944

[14] Sugiyama L 2001 Nucl. Fusion 41739

[15] Garabedian P 2006 Proc. Natl Acad. Sci. USA 10319232

[16] Hirshman S P and Whitson J C 1983 Phys. Fluids 263553

[17] Hirshman S P and Betancourt O 1991 J. Comput. Phys. 9699

[18] Terranova D et al 2010 Plasma Phys. Control. Fusion 52124023

[19] Cooper W A et al 2009 Comput. Phys. Commun. 1801524

[20] Cooper W A, Graves J P, Pochelon A, Sauter O and Villard L 2010 Phys. Rev. Lett. 105035003

[21] Gormezano C et al 2007 Nucl. Fusion 47 S285

[22] Cooper W A, Graves J P and Sauter O 2011 Plasma Phys. Control. Fusion 53024002

[23] Cooper W A et al 2011 Plasma Phys. Control. Fusion 53 at press

[24] Anderson D V, Cooper W A, Gruber R, Merazzi S and Schwenn U 1990 Int. J. Supercomput. Appl. 434

[25] Boozer A H 1980 Phys. Fluids 23904

[26] Nührenberg J and Zille R 1988 Phys. Lett. A 129113

[27] Subbotin A A et al. 2006 Nucl. Fusion 46921

[28] Gobbin M, Spizzo G, Marrelli L and White R B 2010 Phys. Rev. Lett. 105195006 
[29] Cooper W A 1992 Plasma Phys. Control. Fusion 341011

[30] Nührenberg C 1996 Phys. Plasmas 32401

[31] Ahusborde E, Gruber R, Azaiez M and Sawley M L 2007 Phys. Rev. E 75056704

[32] Cooper W A, Fu G Y, Gruber R, Merazzi S, Schwenn U and Anderson D V 1990 Proc. Joint Varenna-Lausane Int. Workshop on Theory of Fusion Plasmas (Varenna, Italy) ed E Sindoni et al (Bologna, Italy: Editrice Compositori) p 655

[33] Nührenberg C (née Schwab) 1993 Phys. Fluids B 53195

[34] Nührenberg C (née Schwab) 1992 Proc. Joint Varenna-Lausanne Int. Workshop on Theory of Fusion Plasmas (Varenna, Italy) ed E Sindoni and J Vaclavik (Bologna, Italy: Editrice Compositori) p 383

[35] Cooper W A 1997 Phys. Plasmas 4153

[36] Piovesan P et al 2009 Nucl. Fusion 49085036

[37] Puiatti M E et al 2009 Plasma Phys. Control. Fusion 51120431

[38] Gobbin M et al 2011 Phys. Rev. Lett. 106025001 